\title{
Rank-one perturbation bounds for singular values of arbitrary matrices
}

\author{
Lei Zhu ${ }^{1}$, Xiaofei Peng ${ }^{2 *}$ (D) and Hao Liü
}

\section{"Correspondence:}

pxf6628@163.com

${ }^{2}$ School of Mathematical Sciences,

South China Normal University,

Guangzhou, P.R. China

Full list of author information is

available at the end of the article

\begin{abstract}
Rank-one perturbation of arbitrary matrices has many practical applications. In this paper, based on the relationship between the singular values and the eigenvalues, we discuss singular value variations and present two-side bounds of the singular values for rank-one perturbation of arbitrary matrices. Numerical results confirm that the proposed perturbation bounds are sharper than some existing bounds.
\end{abstract}

MSC: $65 \mathrm{~F} 15 ; 65 \mathrm{H} 10 ; 65 \mathrm{H} 20$

Keywords: Arbitrary matrix; Singular values; Eigenvalue gap; Rank-one update

\section{Introduction}

Let $\mathcal{C}^{m \times n}\left(\mathcal{C}^{n}\right)$ be the set of $m \times n$ complex matrices ( $n$ dimension vectors). The norm $\|\cdot\|_{2}$ denotes the two norm and the notation $\mathcal{H}(A)=\frac{1}{2}\left(A+A^{*}\right)$ stands for the Hermitian parts of a square matrix $A$. Let $A \in \mathcal{C}^{n \times n}$ have the singular value decomposition (SVD)

$$
A=U \Sigma \mathcal{V}^{*}
$$

where $U \in \mathcal{C}^{n \times n}$ and $\mathcal{V} \in \mathcal{C}^{n \times n}$ are unitary, $\Sigma=\operatorname{diag}\left(\sigma_{1}(A), \ldots, \sigma_{n}(A)\right), \sigma_{i}(A), i=1,2, \ldots, n$, are the singular values of $A$ with

$$
\sigma_{\min }(A)=\sigma_{n}(A) \leq \sigma_{n-1}(A) \leq \cdots \leq \sigma_{1}(A)=\sigma_{\max }(A)
$$

and the superscript $*$ denotes the conjugate transpose.

Singular value variations for rank-one perturbation of arbitrary matrices have many applications, e.g., principal component analysis under a spiked covariance model, and pseudo arc length continuation methods for the solution of systems of nonlinear equations, see [1-4]. Some classical perturbation bounds for singular values can be found in [5], and low rank update of singular values has also been investigated in [6].

In the paper, motivated by the ideas in [7], we consider rank-one perturbation bounds for singular values of arbitrary matrices. Before providing the new bounds, we first introduce the associated results about eigenvalues of Hermitian matrices in [7].

Let $A \in \mathcal{C}^{n \times n}$ be Hermitian and have spectral decomposition

$$
A=V \Lambda V^{*}
$$

(c) The Author(s) 2019. This article is distributed under the terms of the Creative Commons Attribution 4.0 International License (http://creativecommons.org/licenses/by/4.0/), which permits unrestricted use, distribution, and reproduction in any medium, provided you give appropriate credit to the original author(s) and the source, provide a link to the Creative Commons license, and indicate if changes were made. 
where $V=\left(v_{1}, \ldots, v_{n}\right) \in \mathcal{C}^{n \times n}$ is unitary, $\Lambda=\operatorname{diag}\left(\lambda_{1}(A), \ldots, \lambda_{n}(A)\right)$ with the order of its eigenvalues

$$
\lambda_{\min }(A)=\lambda_{n}(A) \leq \cdots \leq \lambda_{1}(A)=\lambda_{\max }(A) .
$$

Define the projection of a vector $x \in \mathcal{C}^{n}$ onto the eigenvectors of a Hermitian matrix $A$ :

$$
x_{i: j} \equiv\left(v_{i}, \ldots, v_{j}\right)^{*} x, \quad i \leq j
$$

Let

$$
\begin{aligned}
& l_{1}(x)=\frac{1}{2}\left(\left\|x_{1: 2}\right\|_{2}^{2}-\operatorname{gap}_{2}+\sqrt{\left(\operatorname{gap}_{2}+\left\|x_{1: 2}\right\|_{2}^{2}\right)^{2}-4 \operatorname{gap}_{2}\left|x_{2}\right|^{2}}\right), \\
& l_{i}(x)=\frac{1}{2}\left(\operatorname{gap}_{i}+\left\|x_{1: i}\right\|_{2}^{2}-\sqrt{\left(\operatorname{gap}_{i}+\left\|x_{1: i}\right\|_{2}^{2}\right)^{2}-4 \operatorname{gap}_{i}\left|x_{i}\right|^{2}}\right), \quad i=2,3, \ldots, n,
\end{aligned}
$$

and for $i=1,2, \ldots, n-1$,

$$
\begin{aligned}
& u_{i}(x)=\frac{1}{2}\left(\left\|x_{i: n}\right\|_{2}^{2}-\operatorname{gap}_{i+1}+\sqrt{\left(\operatorname{gap}_{i+1}+\left\|x_{i: n}\right\|_{2}^{2}\right)^{2}-4 \operatorname{gap}_{i+1}\left\|x_{i+1: n}\right\|_{2}^{2}}\right), \\
& u_{n}(x)=\frac{1}{2}\left(\operatorname{gap}_{n}+\left\|x_{n-1: n}\right\|_{2}^{2}-\sqrt{\left(\operatorname{gap}_{n}+\left\|x_{n-1: n}\right\|_{2}^{2}\right)^{2}-4 \operatorname{gap}_{n}\left|x_{n}\right|^{2}}\right),
\end{aligned}
$$

where $\operatorname{gap}_{i}$ is the distance of an eigenvalue $\lambda_{i}(A)$ to its right neighbor, i.e.,

$$
\operatorname{gap}_{i}=\lambda_{i-1}(A)-\lambda_{i}(A), \quad i=2,3, \ldots, n .
$$

Note that $0 \leq l_{1}(x) \leq\left\|x_{1: 2}\right\|_{2}^{2}, 0 \leq l_{i}(x) \leq\left\|x_{i: n}\right\|_{2}^{2}, i=2, \ldots, n, 0 \leq u_{i}(x) \leq\left\|x_{i: n}\right\|_{2}^{2}, i=$ $1,2, \ldots, n-1$, and $0 \leq u_{n}(x) \leq \operatorname{gap}_{n}$.

With the above notations, the results in [7] are given as follows.

Theorem 1.1 ([7]) Let $A \in \mathcal{C}^{n \times n}$ be Hermitian and $x \in \mathcal{C}^{n}$. Then

$$
\lambda_{i}(A)+l_{i}(x) \leq \lambda_{i}\left(A+x x^{*}\right) \leq \lambda_{i}(A)+u_{i}(x), \quad i=1, n,
$$

and

$$
\lambda_{i}(A)+l_{i}(x) \leq \lambda_{i}\left(A+x x^{*}\right) \leq \min \left\{\lambda_{i}(A)+u_{i}(x), \lambda_{i-1}(A)\right\}, \quad 2 \leq i \leq n-1 .
$$

Notice that the above results improve Weyl's theorem, i.e.,

$$
\begin{aligned}
& \lambda_{i}(A) \leq \lambda_{i}\left(A+x x^{*}\right) \leq \lambda_{i-1}(A), \quad i=2, \ldots, n, \\
& \lambda_{1}(A) \leq \lambda_{1}\left(A+x x^{*}\right) \leq \lambda_{1}(A)+\|x\|_{2}^{2} .
\end{aligned}
$$

In this paper we investigate a singular value case and obtain some singular value variations for rank-one perturbation of arbitrary matrices. The main proof technique is based on Theorem 1.1 and the following relationship:

$$
\sigma_{k}^{2}\left(A+y x^{*}\right)=\lambda_{k}\left(A^{*} A+\|y\|_{2}^{2} x x^{*}+A^{*} y x^{*}+x y^{*} A\right),
$$


where $A \in \mathcal{C}^{n \times n}$ and $x, y \in \mathcal{C}^{n}$. In addition, some existing results on singular values are also used to deduce the new bounds.

\section{Singular value variations}

In this section we present bounds of singular values for rank-one perturbation of arbitrary matrices. We always assume that the singular values and eigenvalues have the decreasing orders given by (1) and (2), respectively.

We first give some notations. Setting $\delta_{k+1}=\sigma_{k}^{2}(A)-\sigma_{k+1}^{2}(A)$. Let

$$
\begin{aligned}
& \zeta_{1}=\frac{1}{2}\left(\|y\|_{2}^{2}\left\|x_{1: 2}\right\|_{2}^{2}-\delta_{2}+\sqrt{\left(\delta_{2}+\|y\|_{2}^{2}\left\|x_{1: 2}\right\|_{2}^{2}\right)^{2}-4 \delta_{2}\|y\|_{2}^{2}\left|x_{2}\right|^{2}}\right), \\
& \zeta_{k}=\frac{1}{2}\left(\delta_{k}+\|y\|_{2}^{2}\left\|x_{1: k}\right\|_{2}^{2}-\sqrt{\left(\delta_{k}+\|y\|_{2}^{2}\left\|x_{1: k}\right\|_{2}^{2}\right)^{2}-4 \delta_{k}\|y\|_{2}^{2}\left|x_{k}\right|^{2}}\right)
\end{aligned}
$$

with $2 \leq k \leq n$,

$$
\phi_{k}=\frac{1}{2}\left(\|y\|_{2}^{2}\left\|x_{k: n}\right\|_{2}^{2}-\delta_{k+1}+\sqrt{\left(\delta_{k+1}+\|y\|_{2}^{2}\left\|x_{k: n}\right\|_{2}^{2}\right)^{2}-4 \delta_{k+1}\|y\|_{2}^{2}\left\|x_{k+1: n}\right\|_{2}^{2}}\right)
$$

with $1 \leq k \leq n-1$,

$$
\phi_{n}=\frac{1}{2}\left(\delta_{n}+\|y\|_{2}^{2}\left\|x_{n-1: n}\right\|_{2}^{2}-\sqrt{\left(\delta_{n}+\|y\|_{2}^{2}\left\|x_{n-1: n}\right\|_{2}^{2}\right)^{2}-4 \delta_{n}\|y\|_{2}^{2}\left|x_{n}\right|^{2}}\right) .
$$

Note that the expressions of $\zeta_{1}, \zeta_{k}, \phi_{k}, \phi_{n}$ are similar to the ones of $l_{1}(x), l_{k}(x), u_{k}(x), u_{n}(x)$, respectively. For simplicity, in the rest of this paper, we also use the notations

$$
\mathcal{S}_{1}=A^{*} A+\|y\|_{2}^{2} x x^{*}, \quad \mathcal{S}_{2}=A^{*} y x^{*}+x y^{*} A .
$$

In order to deduce our results, we give the following lemmas.

Lemma 2.1 ([5]) Let $A, B \in \mathcal{C}^{n \times n}$ be given. Then

$$
\sigma_{i+j-1}(A+B) \leq \sigma_{i}(A)+\sigma_{j}(B)
$$

for $1 \leq i, j \leq n$ and $i+j \leq n+1$.

Lemma 2.2 Let $A \in \mathcal{C}^{n \times n}$ and $x, y \in \mathcal{C}^{n}$. Then

$$
\sigma_{i}\left(y x^{*}\right)=0, \quad i=2, \ldots, n .
$$

Proof The result follows from the fact that $\operatorname{rank}\left(y x^{*}\right) \leq 1$.

Lemma 2.3 Let $A \in \mathcal{C}^{n \times n}$ and $x, y \in \mathcal{C}^{n}$. Then

$$
\begin{aligned}
& \lambda_{1}\left(A^{*} y x^{*}+x y^{*} A\right) \leq 2\left\|A^{*} y x^{*}\right\|_{2}, \\
& \lambda_{n}\left(A^{*} y x^{*}+x y^{*} A\right) \geq-2\left\|A^{*} y x^{*}\right\|_{2} .
\end{aligned}
$$


Proof It is easy to obtain

$$
\lambda_{1}\left(A^{*} y x^{*}+x y^{*} A\right) \leq \sigma_{1}\left(A^{*} y x^{*}+x y^{*} A\right) \leq 2\left\|A^{*} y x^{*}\right\|_{2} .
$$

The lower bound for $\lambda_{n}(*)$ can be obtained by an analogical way.

Lemma 2.4 ([5]) Let $A, B \in \mathcal{C}^{n \times n}$ be Hermitian. Then, for $i=1,2, \ldots, n$, it holds that

$$
\lambda_{k}(A)+\lambda_{n}(B) \leq \lambda_{k}(A+B) \leq \lambda_{k}(A)+\lambda_{1}(B) .
$$

From Lemmas 2.1-2.4 and Theorem 1.1, we can obtain the following bounds of singular values.

Theorem 2.1 Let $A \in \mathcal{C}^{n \times n}$ and $x, y \in \mathcal{C}^{n}$ be given. Then, for $i=1,2, \ldots, n$, it holds that

$$
\max \left\{\sigma_{k+1}(A), L b_{1}, L b_{2}\right\} \leq \sigma_{k}\left(A+y x^{*}\right) \leq \min \left\{\sigma_{k-1}(A), U b_{1}, U b_{2}\right\}
$$

where the two lower bounds $L b_{1}$ and $L b_{2}$ are defined by

$$
L b_{1}=\sigma_{k}(A)-\|x\|_{2}\|y\|_{2}, \quad L b_{2}=\sqrt{\max \left\{0, \sigma_{k}^{2}(A)+\zeta_{k}-2\left\|A^{*} y x^{*}\right\|_{2}\right\}}
$$

and the two upper bounds $U b_{1}$ and $U b_{2}$ are defined by

$$
U b_{1}=\sigma_{k}(A)+\|x\|_{2}\|y\|_{2}, \quad U b_{2}=\sqrt{\sigma_{k}^{2}(A)+\phi_{k}+2\left\|A^{*} y x^{*}\right\|_{2}} .
$$

Here we define $\sigma_{0}(A)=+\infty, \sigma_{n+1}(A)=0 ; \zeta_{k}$, $\phi_{k}$ are given by (4)-(5) and (6)-(7), respectively.

Proof We will complete the proof according to the following three different strategies.

Strategy 1: We use Theorem 1.1 and Lemma 2.3 to deduce the new bounds of $\sigma_{k}\left(A+y x^{*}\right)$. In fact, from (3) and (9), it follows that

$$
\lambda_{k}\left(\mathcal{S}_{1}\right)+\lambda_{n}\left(\mathcal{S}_{2}\right) \leq \sigma_{k}^{2}\left(A+y x^{*}\right) \leq \lambda_{k}\left(\mathcal{S}_{1}\right)+\lambda_{1}\left(\mathcal{S}_{2}\right)
$$

where $\mathcal{S}_{1}$ and $\mathcal{S}_{2}$ are defined by (8). Applying Theorem 1.1 to $\mathcal{S}_{1}$ gives

$$
\sigma_{k}^{2}(A)+\zeta_{k} \leq \lambda_{k}\left(\mathcal{S}_{1}\right) \leq \sigma_{k}^{2}(A)+\phi_{k}
$$

which, together with (13) and Lemma 2.3, yields

$$
\sigma_{k}^{2}(A)+\zeta_{k}-2\left\|A^{*} y x^{*}\right\|_{2} \leq \sigma_{k}^{2}\left(A+y x^{*}\right) \leq \sigma_{k}^{2}(A)+\phi_{k}+2\left\|A^{*} y x^{*}\right\|_{2} .
$$

Strategy 2: We use Lemmas 2.1-2.2 to obtain the new bounds of $\sigma_{k}\left(A+y x^{*}\right)$. In fact, by Lemma 2.2, we have

$$
\sigma_{k_{1}}\left(y x^{*}\right)=0, \quad k_{1}=2,3, \ldots, n .
$$


It follows from Lemma 2.1 that

$$
\sigma_{k+1}(A) \leq \sigma_{k}\left(A+y x^{*}\right)+\sigma_{2}\left(-y x^{*}\right)=\sigma_{k}\left(A+y x^{*}\right), \quad 1 \leq k \leq n-1,
$$

and

$$
\sigma_{k}\left(A+y x^{*}\right) \leq \min _{2 \leq k_{1} \leq k}\left[\sigma_{k+1-k_{1}}(A)+\sigma_{k_{1}}\left(y x^{*}\right)\right]=\sigma_{k-1}(A), \quad 2 \leq k \leq n .
$$

Note that inequalities (15) and (16) also hold for the cases $k=n$ and $k=1$, respectively, based on the definitions $\sigma_{n+1}(A)=0$ and $\sigma_{0}(A)=+\infty$. Combining (15) and (16) gives

$$
\sigma_{k+1}(A) \leq \sigma_{k}\left(A+y x^{*}\right) \leq \sigma_{k-1}(A)
$$

Strategy 3: We use Lemma 2.1 and the definition of the two norm to give the new bounds of $\sigma_{k}\left(A+y x^{*}\right)$. Actually, from Lemma 2.1 and the fact that

$$
\sigma_{1}\left(y x^{*}\right)=\sigma_{1}\left(-y x^{*}\right)=\|x\|_{2}\|y\|_{2},
$$

we have

$$
\begin{aligned}
& \sigma_{k}(A) \leq \sigma_{k}\left(A+y x^{*}\right)+\sigma_{1}\left(-y x^{*}\right)=\sigma_{k}\left(A+y x^{*}\right)+\|x\|_{2}\|y\|_{2}, \\
& \sigma_{k}\left(A+y x^{*}\right) \leq \sigma_{k}(A)+\sigma_{1}\left(y x^{*}\right)=\sigma_{k}(A)+\|x\|_{2}\|y\|_{2},
\end{aligned}
$$

which implies

$$
\sigma_{k}(A)-\|x\|_{2}\|y\|_{2} \leq \sigma_{k}\left(A+y x^{*}\right) \leq \sigma_{k}(A)+\|x\|_{2}\|y\|_{2} .
$$

Now combining bounds (14), (17), (18) and $\sigma_{k}\left(A+y x^{*}\right) \geq 0$, we obtain bounds (10).

Remark 2.1 By Theorem 2.1, the classical bound

$$
\|A+B\|_{2} \leq\|A\|_{2}+\|B\|_{2}
$$

can be improved provided $B$ is of rank one. In fact, from the upper bound of (10), it follows that

$$
\|A+B\|_{2} \leq \sqrt{\|A\|_{2}^{2}+\phi_{1}+2\left\|A^{*} B\right\|_{2}}
$$

which is always sharper than the existing bound $\|A\|_{2}+\|B\|_{2}$ because $\phi_{1} \leq\|B\|_{2}^{2}$.

If we do a restriction on $A^{*} y x^{*}$, then the lower or upper bound of (10) can be further simplified.

Corollary 2.1 Let $A \in \mathcal{C}^{n \times n}$ and $x, y \in \mathcal{C}^{n}$.

(I) If $\mathcal{H}\left(A^{*} y x^{*}\right)$ is positive semidefinite, then the lower bound of (10) is simplified as

$$
\sqrt{\sigma_{k}^{2}(A)+\zeta_{k}} \leq \sigma_{k}\left(A+y x^{*}\right)
$$


(II) If $\mathcal{H}\left(A^{*} y x^{*}\right)$ is negative semidefinite, then the upper bound of (10) is given by

$$
\sigma_{k}\left(A+y x^{*}\right) \leq \min \left\{\sigma_{k-1}(A), \sigma_{k}(A)+\|x\|_{2}\|y\|_{2}, \sqrt{\sigma_{k}^{2}(A)+\phi_{k}}\right\} .
$$

In particular, for the case that $1 \leq k \leq n-1$, the above bound can further be simplified as

$$
\sigma_{k}\left(A+y x^{*}\right) \leq \min \left\{\sigma_{k-1}(A), \sqrt{\sigma_{k}^{2}(A)+\phi_{k}}\right\} .
$$

Proof If $\mathcal{H}\left(A^{*} y x^{*}\right)$ is positive semidefinite, then

$$
\lambda_{n}\left(S_{2}\right)=\lambda_{\min }\left(A^{*} y x^{*}+x y^{*} A\right) \geq 0 .
$$

It follows that the lower bound of (13) is simplified as

$$
\sigma_{k}^{2}\left(A+y x^{*}\right) \geq \lambda_{k}\left(S_{1}\right)
$$

Hence

$$
L b_{2}=\sqrt{\sigma_{k}^{2}(A)+\zeta_{k}} \text {. }
$$

Obviously, for the case, it is easy to check that

$$
\max \left\{\sigma_{k+1}(A), L b_{1}, L b_{2}\right\}=L b_{2},
$$

from which one may deduce bound (19).

If $\mathcal{H}\left(A^{*} y x^{*}\right)$ is negative semidefinite, then

$$
\lambda_{1}\left(S_{2}\right)=\lambda_{\max }\left(A^{*} y x^{*}+x y^{*} A\right) \leq 0
$$

It follows that the upper bound of (13) is simplified as

$$
\sigma_{k}^{2}\left(A+y x^{*}\right) \leq \lambda_{k}\left(S_{1}\right)
$$

Hence

$$
U b_{2}=\sqrt{\sigma_{k}^{2}(A)+\phi_{k}}
$$

which, together with the bounds of (10), gives bound (20). It is noted that $0 \leq \phi_{k} \leq$ $\|y\|_{2}^{2}\left\|x_{k: n}\right\|_{2}^{2}(k=1,2, \ldots, n-1)$. For this case, it is easy to check that

$$
\sqrt{\sigma_{k}^{2}(A)+\phi_{k}} \leq \sigma_{k}(A)+\|x\|_{2}\|y\|_{2}
$$

Therefore, we obtain (21) instead of the upper bound of (10) in Theorem 2.1. This completes the proof. 


\section{Numerical examples}

In this section we give some numerical examples to test the proposed bounds (10) in Theorem 2.1. Numerical examples are carried out in MATLAB R2014b, with machine epsilon $\epsilon \approx 2.2 \times 10^{-10}$.

The first example is randomly generated by MATLAB.

Example 1 Let $A$ be a random matrix of order $n$ and $x, y$ be random vectors of dimension $n$, which can be generated by MATLAB command " $A=\operatorname{randn}(n, n)+1 i * \operatorname{randn}(n, n), x, y=$ $\operatorname{randn}(n, 1)+1 i * \operatorname{randn}(n, 1)$ ". Test bounds $(10)$ in Theorem 2.1 according to the three cases $2 \leq k \leq n-1, k=n$, and $k=1$.

The second example comes from the aero engine fault diagnosis. It is significant to deduce two-side bounds of the singular values for rank-one update of $A$, where $A$ is an available inter-segment attractor reconstruction matrix.

Example 2 Assume that the test signal of the mechanical system is the following numerical sequence: $s_{i}(i=1,2, \ldots, 2 n-1)$, then available inter-segment attractor reconstruction matrix $A$ is

$$
A=\left(\begin{array}{cccc}
s_{1} & s_{2} & \cdots & s_{n} \\
s_{2} & s_{3} & \cdots & s_{n+1} \\
\vdots & \vdots & & \vdots \\
s_{n} & s_{n+1} & \cdots & s_{2 n-1}
\end{array}\right) ;
$$

see $[8,9]$ for more details. The following simulation signals are frequently used in aero engine fault diagnosis. At initial time $t_{0}$ the test signals are described as

$$
s\left(t_{0}\right)=s_{1}+\sigma e\left(t_{0}\right)
$$

After $t$ time, the test signals with engine fault are described as

$$
s(t)=s_{1}+s_{2}+\sigma e(t)
$$

where $s_{1}, s_{2}$ are random signals and $s_{i}(i=3,4, \ldots, 2 n-1)$ are generated in terms of the expression of $s(t)$ at random times; $\sigma e(t)$ is Gaussian white noise with a mean 0 and variance of 1 with $\sigma=1$. Let $x, y$ be random vectors of dimension $n$ and $y x^{*}$ be rank-one update of $A$. Test bounds (10) in Theorem 2.1 according to the three cases $2 \leq k \leq n-1, k=n$, and $k=1$.

In Tables 1-6, we give the lower and upper bounds determined by (10) for the above three cases, which are emphasized by the black text. Note that $\sigma_{n+1}(A)=0$ and $\sigma_{0}(A)=+\infty$. Thus we have omitted the lower bound $\sigma_{n+1}(A)$ in Tables 2 and 5 and the upper bound $\sigma_{0}(A)$ in Tables 3 and 6. For comparison, we also list the exact values of $\sigma_{k}\left(A+y x^{*}\right)$. In addition, for the case of the largest singular values, the existing bounds $\sigma_{1}(A)+\sigma_{1}\left(y x^{*}\right)$ are given. The notations $L b_{i}$ and $U b_{i}(i=1,2)$ are defined by (11) and (12), respectively.

From Tables 1-6 we have the following observations and remarks: 
Table 1 Comparison of the bounds in (10) for Example $1(2 \leq k \leq n-1)$ : the bold face numbers confirm our sharp perturbation bounds

\begin{tabular}{|c|c|c|c|c|}
\hline & & \multicolumn{3}{|l|}{$n=40$} \\
\hline & & $k=8$ & $k=18$ & $k=28$ \\
\hline Lower bounds & $\begin{array}{l}\sigma_{k+1}(A) \\
L b_{1} \\
L b_{2}\end{array}$ & $\begin{array}{l}27.46 \\
\mathbf{2 8 . 5 2} \\
25.60\end{array}$ & $\begin{array}{l}16.34 \\
16.97 \\
\mathbf{1 7 . 6 4}\end{array}$ & $\begin{array}{l}8.41 \\
8.85 \\
7.98\end{array}$ \\
\hline Upper bounds & $\begin{array}{l}\sigma_{k-1}(A) \\
U b_{1} \\
U b_{2}\end{array}$ & $\begin{array}{l}66.30 \\
55.24 \\
\mathbf{4 6 . 3 2}\end{array}$ & $\begin{array}{l}45.10 \\
40.65 \\
\mathbf{3 9 . 1 4}\end{array}$ & $\begin{array}{l}21.46 \\
\mathbf{1 7 . 4 6} \\
19.45\end{array}$ \\
\hline \multirow[t]{3}{*}{ Exact values } & $\sigma_{k}\left(A+y x^{*}\right)$ & 35.23 & 27.35 & 11.46 \\
\hline & & \multicolumn{3}{|l|}{$n=80$} \\
\hline & & $k=38$ & $k=52$ & $k=66$ \\
\hline Lower bounds & $\begin{array}{l}\sigma_{k+1}(A) \\
L b_{1} \\
L b_{2}\end{array}$ & $\begin{array}{l}29.38 \\
\mathbf{3 6 . 4 8} \\
38.20\end{array}$ & $\begin{array}{l}26.19 \\
24.58 \\
\mathbf{2 7 . 3 0}\end{array}$ & $\begin{array}{l}14.57 \\
13.90 \\
\mathbf{1 5 . 0 4}\end{array}$ \\
\hline Upper bounds & $\begin{array}{l}\sigma_{k-1}(A) \\
U b_{1} \\
U b_{2}\end{array}$ & $\begin{array}{l}82.47 \\
\mathbf{8 1 . 6 9} \\
82.41\end{array}$ & $\begin{array}{l}70.06 \\
71.59 \\
\mathbf{6 8 . 3 3}\end{array}$ & $\begin{array}{l}43.35 \\
\mathbf{4 2 . 5 3} \\
45.63\end{array}$ \\
\hline \multirow[t]{3}{*}{ Exact values } & $\sigma_{k}\left(A+y x^{*}\right)$ & 51.24 & 43.45 & 28.24 \\
\hline & & \multicolumn{3}{|l|}{$n=100$} \\
\hline & & $k=16$ & $k=44$ & $k=72$ \\
\hline Lower bounds & $\begin{array}{l}\sigma_{k+1}(A) \\
L b_{1} \\
L b_{2}\end{array}$ & $\begin{array}{l}54.26 \\
\mathbf{5 6 . 1 7} \\
52.46\end{array}$ & $\begin{array}{l}35.34 \\
31.95 \\
\mathbf{3 6 . 3 5}\end{array}$ & $\begin{array}{l}15.85 \\
10.24 \\
\mathbf{1 6 . 3 2}\end{array}$ \\
\hline Upper bounds & $\begin{array}{l}\sigma_{k-1}(A) \\
U b_{1} \\
U b_{2}\end{array}$ & $\begin{array}{l}93.14 \\
\mathbf{8 6 . 2 0} \\
89.35\end{array}$ & $\begin{array}{l}75.35 \\
70.28 \\
78.01\end{array}$ & $\begin{array}{l}44.52 \\
43.13 \\
48.76\end{array}$ \\
\hline Exact values & $\sigma_{k}\left(A+y x^{*}\right)$ & 70.56 & 45.15 & 30.42 \\
\hline
\end{tabular}

Table 2 Comparison of the bounds in (10) for smallest singular values of Example 1: the bold face numbers confirm our sharp perturbation bounds

\begin{tabular}{lllll}
\hline \multirow{2}{*}{ Lower bounds } & & \multicolumn{3}{l}{$n$} \\
\cline { 3 - 5 } & & 60 & 90 & 120 \\
\hline \multirow{3}{*}{ Upper bounds } & $L b_{1}$ & 11.63 & 20.14 & $\mathbf{1 8 . 9 0}$ \\
& $\sigma_{n-1}(A)$ & 21.05 & 41.64 & 38.60 \\
& $U b_{1}$ & $\mathbf{2 0 . 8 3}$ & $\mathbf{3 9 . 8 3}$ & 34.53 \\
Exact values & $U b_{2}$ & 24.47 & 43.08 & $\mathbf{3 3 . 6 4}$ \\
\hline
\end{tabular}

Table 3 Comparison results of the bounds for largest singular values of Example 1: the bold face numbers confirm our sharp perturbation bounds

\begin{tabular}{|c|c|c|c|c|}
\hline & & \multicolumn{3}{|l|}{$n$} \\
\hline & & 20 & 40 & 60 \\
\hline Lower bounds & $\begin{array}{l}\sigma_{2}(A) \\
L b_{1} \\
L b_{2}\end{array}$ & $\begin{array}{l}18.46 \\
19.68 \\
\mathbf{2 1 . 8 4}\end{array}$ & $\begin{array}{l}45.26 \\
23.89 \\
\mathbf{4 6 . 9 0}\end{array}$ & $\begin{array}{l}37.64 \\
32.70 \\
\mathbf{4 0 . 0 2}\end{array}$ \\
\hline Upper bounds & $\begin{array}{l}U b_{1} \\
U b_{2} \\
\sigma_{1}(A)+\sigma_{1}\left(y x^{*}\right)\end{array}$ & $\begin{array}{l}50.68 \\
\mathbf{4 2 . 0 4} \\
58.53\end{array}$ & $\begin{array}{l}72.39 \\
65.79 \\
86.48\end{array}$ & $\begin{array}{l}81.06 \\
73.80 \\
96.46\end{array}$ \\
\hline Exact values & $\sigma_{1}\left(A+y x^{*}\right)$ & 30.58 & 69.57 & 48.19 \\
\hline
\end{tabular}


Table 4 Comparison of the bounds in (10) for Example $2(2 \leq k \leq n-1)$ : the bold face numbers confirm our sharp perturbation bounds

\begin{tabular}{lllll}
\hline & & $n=30$ & & \\
\cline { 3 - 5 } & & $k=8$ & $k=16$ & $k=24$ \\
\hline Lower bounds & $\sigma_{k+1}(A)$ & 16.25 & 12.75 & 26.81 \\
& $L b_{1}$ & 13.96 & $\mathbf{1 2 . 9 1}$ & $\mathbf{2 8 . 5 4}$ \\
& $L b_{2}$ & $\mathbf{1 7 . 1 4}$ & 10.27 & 19.72 \\
Upper bounds & $\sigma_{k-1}(A)$ & 53.45 & 36.08 & 51.08 \\
& $U b_{1}$ & 45.26 & $\mathbf{3 4 . 7 7}$ & $\mathbf{4 8 . 7 6}$ \\
& $U b_{2}$ & $\mathbf{4 0 . 1 8}$ & 34.82 & 50.73 \\
Exact values & $\sigma_{k}\left(A+y x^{*}\right)$ & 34.03 & 27.59 & 48.50 \\
\hline \hline & & $n=60$ & & \\
\cline { 3 - 5 } & & $k=22$ & $k=40$ & $k=58$ \\
\hline Lower bounds & $\sigma_{k+1}(A)$ & 30.13 & 23.26 & 10.26 \\
& $L b_{1}$ & 32.53 & $\mathbf{2 5 . 1 1}$ & 14.96 \\
& $L b_{2}$ & $\mathbf{3 6 . 1 8}$ & 10.04 & $\mathbf{1 5 . 6 0}$ \\
Upper bounds & $\sigma_{k-1}(A)$ & 68.26 & 60.29 & 49.07 \\
& $U b_{1}$ & 70.24 & 58.65 & 47.43 \\
& $U b_{2}$ & $\mathbf{6 8 . 0 1}$ & $\mathbf{5 4 . 7 1}$ & $\mathbf{4 5 . 4 8}$ \\
Exact values & $\sigma_{k}\left(A+y x^{*}\right)$ & 56.02 & 41.59 & 28.56 \\
\hline
\end{tabular}

Table 5 Comparison of the bounds in (10) for smallest singular values of Example 2: the bold face numbers confirm our sharp perturbation bounds

\begin{tabular}{lllll}
\hline & & $n$ & & \\
\cline { 3 - 5 } & & 28 & 62 & 96 \\
\hline Lower bounds & $L b_{1}$ & $\mathbf{2 4 . 1 4}$ & 16.84 & $\mathbf{1 1 . 4 8}$ \\
& $L b_{2}$ & 22.03 & $\mathbf{1 7 . 0 6}$ & 10.05 \\
Upper bounds & $\sigma_{n-1}(A)$ & 38.14 & 39.23 & 34.29 \\
& $U b_{1}$ & $\mathbf{3 2 . 8 0}$ & 38.10 & $\mathbf{3 0 . 4 0}$ \\
& $U b_{2}$ & 34.28 & $\mathbf{3 7 . 9 5}$ & 37.22 \\
Exact values & $\sigma_{n}\left(A+y x^{*}\right)$ & 28.55 & 27.83 & 17.74 \\
\hline
\end{tabular}

Table 6 Comparison results of the bounds for largest singular values of Example 2: the bold face numbers confirm our sharp perturbation bounds

\begin{tabular}{lllll}
\hline & & $n$ & & \\
\cline { 3 - 5 } & & 20 & 40 & 60 \\
\hline Lower bounds & $\sigma_{2}(A)$ & 16.18 & 40.04 & 32.54 \\
& $L b_{1}$ & 17.05 & 28.60 & 30.11 \\
& $L b_{2}$ & $\mathbf{2 0 . 5 3}$ & $\mathbf{4 0 . 0 8}$ & $\mathbf{3 6 . 2 9}$ \\
Upper bounds & $U b_{1}$ & 46.13 & 68.25 & $\mathbf{7 0 . 5 7}$ \\
& $U b_{2}$ & $\mathbf{4 0 . 1 6}$ & $\mathbf{6 0 . 2 7}$ & 76.90 \\
& $\sigma_{1}(A)+\sigma_{1}\left(y x^{*}\right)$ & 48.79 & 69.47 & 84.18 \\
Exact values & $\sigma_{1}\left(A+y x^{*}\right)$ & 27.57 & 53.13 & 49.27 \\
\hline
\end{tabular}

(1) From comparison results with the exact values, the proposed bounds (10) are feasible and effective.

(2) For Examples 1-2, the lower and the upper bounds given by (10) are chosen from $L b_{1}, L b_{2}$ and $U b_{1}, U b_{2}$ instead of $\sigma_{k+1}(A)$ and $\sigma_{k-1}(A)$, respectively. In other words, the bounds $L b_{i}(i=1,2)$ and $U b_{i}(i=1,2)$ are sharper than $\sigma_{k+1}(A)$ and $\sigma_{k-1}(A)$, 
respectively. Note that $\sigma_{k+1}(A)$ and $\sigma_{k-1}(A)$ are essentially the existing bounds given by Lemma 2.1. This further verifies the advantage of the proposed bounds.

(3) In particular, from Tables 3 and 6, the proposed upper bounds of (10) are always tighter than the existing upper bounds $\sigma_{1}(A)+\sigma_{1}\left(y x^{*}\right)$, which agrees with Remark 2.1.

\section{Conclusions}

In this paper, by making use of different strategies, we present the two-side bounds of singular values for rank-one perturbation of arbitrary matrices. In particular, the proposed upper bounds are proved to be always sharper than the classical bound $\|A+B\|_{2} \leq\|A\|_{2}+$ $\|B\|_{2}$. Numerical examples further demonstrate the feasibility and effectiveness of the new perturbation bounds, which are tighter than some existing perturbation bounds.

\section{Acknowledgements}

The authors would like to thank the anonymous referees for their valuable comments and suggestions, which greatly improved the paper.

\section{Funding}

The work was supported by the National Natural Science Foundation of China under Grants Nos. U1533202, U1811464, 11571124, 11671158, 11801097, the Jiangsu Provincial Natural Science Foundation of Jiangsu Province of China under Grant No. BK20181405, and Civil Aviation Science and Technology Project under Grant No. 20150218.

\section{Availability of data and materials}

The datasets generated during and/or analysed during the current study are available from the corresponding author on reasonable request.

\section{Competing interests}

The authors declare that they have no competing interests.

Authors' contributions

All authors contributed equally to this work. All authors read and approved the final manuscript.

\section{Author details}

${ }^{1}$ College of Engineering, Nanjing Agricultural University, Nanjing, P.R. China. ${ }^{2}$ School of Mathematical Sciences, South China Normal University, Guangzhou, P.R. China. ${ }^{3}$ College of Science, Nanjing University of Aeronautics and Astronautics, Nanjing, P.R. China.

\section{Publisher's Note}

Springer Nature remains neutral with regard to jurisdictional claims in published maps and institutional affiliations.

Received: 26 February 2019 Accepted: 2 May 2019 Published online: 14 May 2019

\section{References}

1. Dickson, K.I., Kelley, C.T., Ipsen, I.C.F., Kevrekidis, I.G.: Condition estimation for pseudo-arclength continuation. SIAM J. Numer. Anal. 45(1), 263-276 (2007)

2. Xu, W.W., Ma, L.J., Zhu, L., Liu, H.: On interval estimates of perturbations of generalized eigenvalues for diagonalizable pairs. Linear Algebra Appl. 562, 15-43 (2019)

3. Xu, W.W., Pang, H.K., Li, W., Huang, X.P., Guo, W.-J.: On the explicit expression of chordal metric between generalized singular values of Grassmann matrix pairs with applications. SIAM J. Matrix Anal. Appl. 39(4), 1547-1563 (2018)

4. Xu, W.W., Li, W., Zhu, L., Huang, X.P.: The analytic solutions of constrained matrix minimization and maximization problems and its applications. SIAM J. Optim. (2019, accepted)

5. Horn, R.A., Johnson, C.R.: Topics in Matrix Analysis. Cambridge University Press, London (1991)

6. Chu, D.L., Chu, M.: Low rank update of singular values. Math. Comput. 75(255), 1351-1366 (2006)

7. Ipsen, I.C.F., Nadler, B.: Refined perturbation bounds for eigenvalues of Hermitian and non-Hermitian matrices. SIAM J. Matrix Anal. Appl. 31, 40-53 (2009)

8. Sun, J.Z., Zuo, H.F., Liu, P.P., Zhu, L.: A method of condition monitoring and on-wing life prediction for civil aviation aircraft engine based on dynamic linear model. IEEE Trans. Reliab. 33(12), 3243-3250 (2013)

9. Zhu, L., Zuo, H.F., Cai, J.: Performance reliability prediction for civil aviation aircraft engine based on Wiener process. J. Aerosp. Power 28(5), 1006-1012 (2013) 